\title{
Haplotype divergence and multiple candidate genes at $R p h q 2$, a partial resistance QTL of barley to Puccinia hordei
}

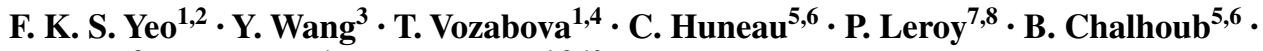 \\ X. Q. $\mathbf{Q i}^{3} \cdot$ R. E. Niks ${ }^{1} \cdot$ T. C. Marcel ${ }^{1,9,10}$
}

Received: 19 January 2015 / Accepted: 17 October 2015 / Published online: 5 November 2015

(C) The Author(s) 2015. This article is published with open access at Springerlink.com

\begin{abstract}
Key message Rphq2, a minor gene for partial resistance to Puccinia hordei, was physically mapped in a 188 kbp introgression with suppressed recombination between haplotypes of $r p h q 2$ and $R p h q 2$ barley cultivars.

Abstract Partial and non-host resistances to rust fungi in barley (Hordeum vulgare) may be based on pathogenassociated molecular pattern (PAMP)-triggered immunity. Understanding partial resistance may help to understand non-host resistance, and vice versa. We constructed two non-gridded BAC libraries from cultivar Vada and line SusPtrit. Vada is immune to non-adapted Puccinia rust fungi, and partially resistant to $P$. hordei. SusPtrit is
\end{abstract}

Communicated by B. Keller.

Electronic supplementary material The online version of this article (doi:10.1007/s00122-015-2627-5) contains supplementary material, which is available to authorized users.

\section{R. E. Niks}

rients.niks@wur.nl

1 Laboratory of Plant Breeding, Wageningen University, Droevendaalsesteeg 1, 6708PB, 6700 AJ Wageningen, The Netherlands

2 Present Address: Department of Plant Science and Environmental Ecology, Faculty of Resource Science and Technology, University Malaysia Sarawak, 94300 Kota Samarahan, Sarawak, Malaysia

3 Key Laboratory of Plant Molecular Physiology, Institute of Botany, Chinese Academy of Sciences, Nanxincun 20, Fragrant Hill, Beijing 100093, China

4 Present Address: The Institute of Botany of the Academy of Science of the Czech Republic, Zámek 1, 252

43 Průhonice, Czech Republic susceptible to several non-adapted rust fungi, and has been used for mapping QTLs for non-host and partial resistance. The BAC libraries help to identify genes determining the natural variation for partial and non-host resistances of barley to rust fungi. A major-effect QTL, Rphq2, for partial resistance to $P$. horde $i$ was mapped in a complete Vada and an incomplete SusPtrit contig. The physical distance between the markers flanking Rphq2 was $195 \mathrm{Kbp}$ in Vada and at least $226 \mathrm{Kbp}$ in SusPtrit. This marker interval was predicted to contain 12 genes in either accession, of which only five genes were in common. The haplotypes represented by Vada and SusPtrit were found in 57 and $43 \%$, respectively, of a 194 barley accessions panel. The lack of homology between the two haplotypes probably explains the suppression of recombination in the Rphq2 area and limit further genetic resolution in fine mapping. The possible candidate genes for $R p h q 2$ encode peroxidases, kinases and a member of seven-in-absentia protein family. This result suggests that $R p h q 2$ does not belong to the NB-LRR

5 INRA, UMR1165, Unité de Recherche en Génomique Végétale, 91057 Evry, France

6 Université d'Evry Val d'Essonne, UMR1165, Unité de Recherche en Génomique Végétale, 91057 Evry, France

7 INRA, UMR1095, Genetics Diversity and Ecophysiology of Cereals, 63039 Clermont-Ferrand, France

8 Université Blaise Pascal, UMR1095, Genetics Diversity and Ecophysiology of Cereals, 63039 Clermont-Ferrand, France

9 INRA, UMR1290, BIOGER, 78850 Thiverval-Grignon, France

10 AgroParisTech, UMR1290, BIOGER, 78850 Thiverval-Grignon, France 
gene family and does not resemble any of the partial resistance genes cloned previously.

\section{Introduction}

Partial resistance is an incomplete host resistance which retards epidemic development despite a compatible infection type (Niks et al. 2011; Parlevliet and van Ommeren 1975). Partial resistance of barley to Puccinia hordei consists of the components lower infection frequency, lower sporulation rate and longer latency period of the pathogen, and such effects are not associated with hypersensitivity. Evidence suggests partial resistance of barley to powdery mildew and rust fungi to be a weak form of non-host resistance based on pathogen-associated molecular pattern (PAMP)-triggered immunity (PTI) (Jones and Dangl 2006; Niks and Marcel 2009; Niks et al. 2011; Trujillo et al. 2004). Non-host resistance is, by definition, a resistance observed in all genotypes of a plant species to all genotypes of a potential pathogen species (Niks et al. 2011). However, plant species may turn out to have a marginal host or near-non-host status to some pathogen species (Niks 1987). Barley (Hordeum vulgare) is such a marginal host or near-non-host to Puccinia triticina, Puccinia hordei-murini and some other non-adapted rust fungi because a small proportion of barley accessions (less than $10 \%$ ) are susceptible (>10 pustules on the first leaf) at seedling stage when inoculum is applied at high density (Atienza et al. 2004). In the context of this paper, non-adapted rust fungi are rust fungi that are poorly or not adapted to barley, but primarily to one or few other plant species. We try to investigate non-host resistance by dissecting the genetics of near-non-host resistance with the assumption that we can extrapolate the findings to explain also the basis of full non-host resistance. SusPtrit was developed for such a purpose by accumulating susceptibility genes from rare barley accessions that were somewhat susceptible to $P$. triticina at the seedling stage. SusPtrit is, at the seedling stage, highly susceptible to P. triticina and to at least nine other non-adapted rust fungi (Atienza et al. 2004). This experimental line was used to develop mapping populations Vada/SusPtrit (Jafary et al. 2006), Cebada Capa/SusPtrit (Jafary et al. 2008) and SusPtrit/Golden Promise (Yeo et al. 2014), as well as a set of near-isogenic lines for different resistances QTLs (Yeo et al. unpublished data). Resistance of barley to non-adapted rust fungi inherits polygenically just as partial resistance to $P$. hordei, the barley leaf rust fungus (Jafary et al. 2006, 2008; Marcel et al. 2007b; Niks et al. 2000; Qi et al. 1998).

The locations of genes for resistance to non-adapted rust fungi tend to overlap with those for partial resistance to P. hordei (González et al. 2010; Jafary et al. 2008; Niks and Marcel 2009) and they share the same prehaustorial resistance mechanism, viz. a high rate of failed attempt to haustorium formation (Niks 1983a, b). If both the partial resistance to adapted fungi and non-host resistance to nonadapted fungi are mainly based on PTI, a better understanding of partial resistance will help us to gain more insight into non-host resistance, and vice versa. More than 20 partial resistance quantitative trait loci (QTLs) to $P$. hordei have been mapped at seedling and adult plant stages (Jafary et al. 2006; Marcel et al. 2007b, 2008; Niks et al. 2000; Qi et al. 1998, 1999). To determine the underlying genes of those QTLs, we need to identify and validate the candidate genes of a selection of QTLs. To date, five large-effect resistance QTLs have been cloned (Fu et al. 2009; Fukuoka et al. 2009; Hayashi et al. 2010; Krattinger et al. 2009; Manosalva et al. 2009). The cloned QTLs are different from each other in gene structure and function, which implies a diversity of mechanisms underlying partial resistance. The genes that have been cloned so far do not have the typical modular NB-LRR structure of $R$-genes. Rphq2 is one of the partial resistance QTLs to $P$. hordei mapped at the telomeric region of chromosome 2HL in L94/Vada (Qi et al. 1998), Vada/SusPtrit (Jafary et al. 2006) and in an association mapping study comprising 146 barley genotypes (Kraakman et al. 2006). In L94/ Vada and Vada/SusPtrit populations, Vada is the donor of Rphq2. Vada is a cultivar developed from Hordeum laevigatum/Gold (Dros 1957). Near-isogenic lines (NILs) are available for Rphq2 in L94 genetic background (L94Rphq2) (Marcel et al. 2007a; van Berloo et al. 2001) and for rphq2 of L94 in Vada background (Vada-rphq2) (Marcel et al. 2007a). L94 is an Ethiopian landrace-derived line that has some level of susceptibility to some non-adapted rust fungi (e.g., Puccinia triticina, P. hordei-secalini and P. hordei-murini) (Atienza et al. 2004; Niks 1983a). When L94-Rphq2 was inoculated with $P$. hordei-secalini and $P$. hordei-murini, it had a significantly lower infection level compared to L94 (Yeo et al. unpublished data). This suggests that the postulated $R p h q 2$ gene explaining the resistance to $P$. hordei also affects the resistance to some non-adapted rust species. Recently, Johnston et al. (2013) suggested that $R p h q 2$ is possibly a weaker allelic form of a novel leaf rust resistance gene Rph22 (or Rph22.ak) found in $H$. bulbosum, a non-host species for P. hordei. Similar to Rphq2, Rph22 confers a non-hypersensitive reaction resistance. Therefore, it is interesting to clone $R p h q 2$ which will provide molecular information to further study partial and non-host resistances, and their possible association. The high-resolution mapping of Rphq2 allowed pinpointing this QTL to an interval of about $0.1 \mathrm{cM}$, corresponding to about 121-198 kb (Marcel et al. 
2007a). This estimated physical interval is sufficiently small to justify the development of a Bacterial Artificial Chromosomes (BAC) library to pin down Rphq2 to one or few candidate genes.

Construction and organization of BAC libraries remains laborious and costly, especially for organisms with large and complex genomes like barley [4.98 Gb (The International Barley Genome Sequencing Consortium 2012)]. In barley, about 200,000 clones with an average insert size of $120 \mathrm{~kb}$ would be required to achieve a genome coverage of five genome-equivalents, which is needed for a more than $99 \%$ probability of recovering any specific sequence of interest. To date, gridded BAC libraries are available for barley cv. Morex (Schulte et al. 2011; Yu et al. 2000), Haruna Nijo (Saisho et al. 2007) and a doubled haploid barley line CS134 derived from Clipper/Sahara-3771 (Shi et al. 2010). The inconveniences linked to the gridding, storage and maintenance of such a quantity of clones can be circumvented by the pooled library approach described by Ma et al. (2000) for wheat and Isidore et al. (2005) for barley. This approach consists of pooling several hundreds of clones together without the need of picking and storing individual clones. The first pooled BAC library of barley developed from cv. Cebada Capa was successfully used to establish a single contig of six BAC clones spanning $230 \mathrm{~kb}$ at the $R p h 7$ locus on chromosome $3 \mathrm{H}$ (Isidore et al. 2005). The BAC libraries from the four mentioned barley genotypes could help in the construction of physical maps around any target gene, but to isolate genes of interest in plants, it is often essential to construct BAC libraries from specific genotypes. Indeed, the gene content may vary between individuals of the same species and the gene of interest may not be present in the genomic libraries of conspecific accessions. This consideration is especially relevant concerning the genes involved in resistance to pathogens, which are known to be under strong selective pressure (Meyers et al. 2005; Salvaudon et al. 2008; Shen et al. 2006).

The current study aimed to develop two non-gridded BAC libraries from cultivar Vada and line SusPtrit, which will allow the isolation of genes for partial and non-host resistances. Having a BAC library from the resistant parent as well as from a susceptible parent is required because genes involved in such resistances can either be a resistance or susceptibility factor. After screening the newly developed BAC libraries, we identified and sequenced BAC clones in the Rphq2 region of both genotypes. The assembly and annotation of BAC sequences revealed a major divergence in haplotypes at the Rphq2 locus and several genes that might be responsible for the phenotypic contrast between Vada and SusPtrit for partial resistance due to Rphq2.

\section{Materials and methods}

\section{Construction of two non-gridded BAC libraries from Vada and SusPtrit}

We constructed two non-gridded BAC libraries of barley from the cv. Vada and from the experimental line SusPtrit, respectively. Vada not only carries the resistance allele of our target QTL for map-based cloning, Rphq2, but also many other QTLs for partial and non-host resistances to adapted and non-adapted rust species (Jafary et al. 2006, 2008), for which SusPtrit contains the susceptibility allele.

The methodology followed to construct the two BAC libraries has been described in detail by Peterson et al. (2000), with several modifications proposed in subsequent reports (Allouis et al. 2003; Chalhoub et al. 2004; Isidore et al. 2005). A detailed description of the methodology to construct the BAC libraries of Vada and SusPtrit is available as supplementary material (Supplemental Material 1). Briefly, high molecular weight (HMW) DNA was isolated from 40 to $50 \mathrm{~g}$ of 2-4-week-old leaves. Plugs of HMW DNA were partially digested with the HindIII restriction enzyme. Partially digested DNA was subjected to a single size selection and fractions of DNA fragments were recovered from agarose plugs in the ranges $50-100 \mathrm{~kb}$ (H0 fraction), $100-150 \mathrm{~kb}$ (H1 fraction), $150-200 \mathrm{~kb}$ (H2 fraction), 200-250 kb (H3 fraction) and 250-300 (H4 fraction). The insert DNA from $\mathrm{H} 0$ to $\mathrm{H} 4$ fractions was ligated separately into the pIndigoBAC vector (CalTech) prepared for highefficiency cloning with HindIII as described by Chalhoub et al. (2004) or into the commercial pIndigoBAC-5 vector (Epicentre Biotechnologies). BAC clones were obtained by electroporation of the de-salted ligation mixtures into ElectroMax DH10B electrocompetent cells (Invitrogen). Pools of BAC clones were prepared by collecting colonies on plates calibrated to contain an average of about 1500 colonies per plate, as suggested by Isidore et al. (2005). The pools were then aliquoted into four tubes, each corresponding to one copy of the library (copies A, B, C and D). Copy A is stored in a $-80^{\circ} \mathrm{C}$ freezer at Unité de Recherche en Génomique Végétale (URGV, Evry, France), copy B at Institute of Botany, Chinese Academy of Sciences (IBCAS, Beijing, China). Copies $\mathrm{C}$ and $\mathrm{D}$ are stored at Wageningen UR, Plant Breeding (WUR, the Netherlands).

\section{Characterization of the BAC libraries}

Twenty-four BAC clones were randomly selected from the fractions $\mathrm{H} 1, \mathrm{H} 2$ and $\mathrm{H} 3$ of each library (i.e., $72 \mathrm{BAC}$ clones per library) and grown for $24 \mathrm{~h}$ at $37{ }^{\circ} \mathrm{C}$ in $1.5 \mathrm{ml}$ LB medium containing $12.5 \mu \mathrm{g}$ CAM. The BAC DNA was extracted following an alkaline lysis procedure (Sambrook 
et al. 1989) with ready-to-use buffers P1, P2 and P3 (Qiagen) and digested overnight with NotI (New England Biolabs). Digested products were separated on a $1 \%$ SeaKem $^{\circledR}$ LE agarose gel (BMA) in $0.5 \times$ TBE in a CHEF-DR ${ }^{\mathrm{TM}}$ II apparatus (Bio-Rad) with the following pulsed field gel electrophoresis parameters: $200 \mathrm{~V}, 5-15$ s switch time, for $14.3 \mathrm{~h}$ at $10{ }^{\circ} \mathrm{C}$. The insert sizes of selected BAC clones were estimated after comparison with the CHEF DNA size standard lambda ladder (Bio-Rad) run in the same gel.

BAC pool DNA was isolated from $250 \mu \mathrm{l}$ aliquot per pool from the copy D of the libraries as described previously. The two barley BAC libraries were characterized for genome representation by PCR screening of 46 pools per library with one microsatellite marker from each of the 14 barley chromosome arms. The markers were selected from the barley microsatellite consensus map of Varshney et al. (2007). Robust markers that were polymorphic between Vada and SusPtrit were selected in priority. For only two microsatellites, GBMS062 and GBM1482, Vada and SusPtrit had the same allele. The number of positive pools was determined by counting the number of pools displaying a band of similar size as the one of the parental genomic DNA run on the same gel. None of the bands amplified in a BAC pool from one genotype had the size of the allele from the other genotype, indicating that contamination of one library with clones from the other library is unlikely. The reverse primer of each microsatellite was labeled with IRDye-700 or IRDye-800 and the PCR product visualized on a LICOR 4200 DNA sequencer (LICOR ${ }^{\circledR}$ Biosciences).

\section{Identification of BAC clones spanning the Rphq2 locus}

The work flow for identifying Vada and SusPtrit BAC clones spanning the Rphq2 locus is presented in Supplemental Fig. 1. The BAC libraries were screened following a PCR-based method. In a first step, to screen the BAC pools and to identify positive BAC clones, we used molecular markers known to be closely linked to Rphq2 (Marcel et al. 2007a; Table 1). In a second step, after the identification and sequencing of several positive BAC clones, we used primers designed to amplify the BAC end sequences (bes), the genes annotated in these clones and newly developed markers. We also used primers to amplify sequences at the edges of gaps in the already assembled BAC sequences (Table 1). Positive single-BAC clones were validated after PCR amplification with the markers and primers used during the screening process and maintained in glycerol stock (LB supplemented with $25 \%$ glycerol). The insert size of the BAC clones was determined as described in the previous section before further analyses. The BAC clones were named as follows: "BAC pool number; Sub-pool number (384-well plate number); row letter; column number; single-colony letter" (e.g., V41 $\underline{\text { P7 }} \underline{\mathrm{L}} \underline{3} \underline{\mathrm{A}}$ ).
All the confirmed positive BAC clones from the Vada and SusPtrit libraries were fingerprinted and their extremities sequenced. BAC clone fingerprints were obtained following the AFLP procedure from Brugmans et al. (2006) using the HindIII/TaqI restriction enzyme combination. The generated fragments were separated on a LICOR 4200 DNA sequencer (LICOR Biosciences, Lincoln, NE, USA). Shared bands between BAC clones indicated sequence overlaps between the clones. BAC end sequences were obtained following a procedure consisting of a nested PCR approach on restriction digests of BAC clones DNA ligated with specific adaptors (Supplemental Material 1). Primers which amplify the BAC ends were designed using Lasergene software (DNASTAR ${ }^{\circledR} 8$ Inc., Madison, WI, USA).

\section{BAC clone sequencing and annotation}

The Rphq2 genetic window is delimited by proximal (WBE114) and distal (WBE115) markers for which at least one recombination event has been found with $R p h q 2$. Three BAC clones which fully covered the Rphq2 genetic window (V41P7L3A, V48P5B18A and V76P5D5A) were sequenced following a Sanger approach at Macrogen. One SusPtrit BAC clone was sequenced via a Sanger approach as well, S35P2K14E. The number of reads obtained for the BAC clones of Vada and SusPtrit ranges from 1000 to 1740 reads with an average length of $867 \mathrm{bp}$, corresponding to $8 \times$ sequencing depths. For each BAC clone, the short Sanger sequences were assembled in contigs and ordered by Macrogen. Several gaps remained within the BAC clones with 5-14 contigs per clone. Therefore, the three BAC clones of Vada and S35P2K14E of SusPtrit were re-sequenced following a 454 sequencing approach at Greenomics ${ }^{\mathrm{TM}}$ to bridge the gaps. Two additional BAC clones of SusPtrit (S35P1J10A and S73P5N20A) were also sequenced following this 454 sequencing approach. The three SusPtrit BAC clones, S35P2K14E with S35P1J10A and S73P5N20A, together cover only partially the targeted Rphq2 genetic window. The number of reads per BAC clone obtained from 454 sequencing ranges from 15,570 to 38,120 reads with an average length of $350 \mathrm{bp}$, corresponding to $20 \times$ sequencing depths for each BAC clone. For each BAC clone, the short sequences obtained by 454 sequencing were assembled in contigs by Greenomics ${ }^{\mathrm{TM}}$.

The obtained 454 sequence contigs were aligned to the previously assembled Sanger sequence contigs [Lasergene software (DNASTAR ${ }^{\circledR} 8$ Inc., Madison, WI, USA)]. No discrepancy was observed between the assemblies obtained via both approaches. Based on the AFLP fingerprints of the BAC clones of Vada, V76P5D5A overlaps proximally with V41P7L3A and distally with V48P5B18A. We identified the overlapping sequences and confirmed the fingerprints. According to the AFLP fingerprints of the BAC 


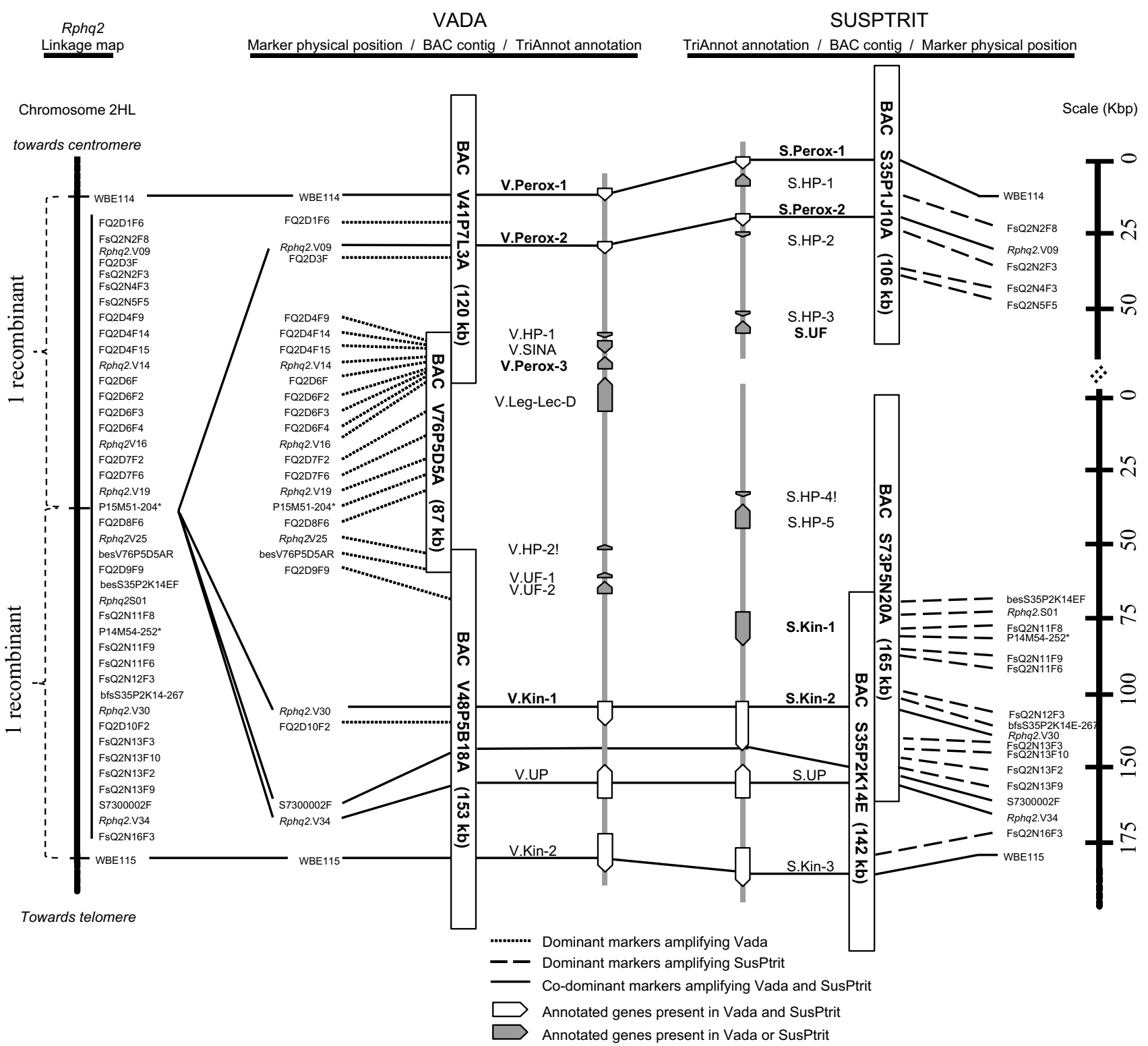

Fig. 1 Representation of the $0.1 \mathrm{cM}$ marker interval between WBE114 and WBE115 containing Rphq2: including the linkage map, the BAC contig of Vada and SusPtrit, and the genes annotated with the TriAnnot pipeline. The size of the BAC clones was estimated by NotI digestion. The genes followed by ! have been predicted only by the TriAnnot pipeline and not by RiceGAAS. The genes in bold were supported by sequences from the transcriptomes of Vada and L94,

clones of SusPtrit, S73P5N20A overlaps with S35P2K14E, and S35P1J10A is located proximally but is not overlapping. We identified the overlapping sequences between S73P5N20A and S35P2K14E which also helped us to order the 454 sequence contigs. The 454 sequence contigs obtained for clone S35P1J10A were ordered according to a dot plot analysis against V41P7L3A which shares the same marker (WBE114). The dot plot analysis was carried out using MUMmer software (Kurtz et al. 2004). indicating that they are expressed in the leaves. Markers followed by * are AFLP-converted single locus PCR markers developed by Marcel et al. (2007a). HP hypothetical protein, Kin kinase, Leg-Lec-D legume lectin domain, Perox peroxidase, $S$ SusPtrit, SINA seven-inabsentia protein, $U F$ unknown function, $U P$ uncharacterized protein, $V$ Vada

The consensus sequence corresponding to the Rphq2 genetic window of Vada, flanked by markers WBE114 and WBE115, was generated and annotated using the TriAnnot pipeline version 3.5 (Leroy et al. 2012) following the barley default analysis template (http://clermont.inra.fr/triannot). The consensus sequence corresponding to the Rphq2 genetic window of SusPtrit was annotated separately using the same pipeline, TriAnnot_v3.5. The sequences of Vada and SusPtrit were also annotated separately using 
Table 1 Primer pairs used to identify BAC clones of Vada and SusPtrit at the Rphq2 locus

\begin{tabular}{|c|c|c|}
\hline Name & Primers sequences $\left(5^{\prime}-3^{\prime}\right)$ & $T_{\mathrm{a}}\left({ }^{\circ} \mathrm{C}\right)$ \\
\hline${ }^{\mathrm{M}} \mathrm{WBE} 114^{V S}$ & $\begin{array}{l}F: \text { GGCGACCTCCAGCGTATC } \\
R: \text { GTGGTTCGGTCCTTGATGAG }\end{array}$ & 58 \\
\hline${ }^{\mathrm{M}} \mathrm{WBE} 115^{V S}$ & $\begin{array}{l}F: \text { GGCGGTCGGCATCGTCCAGT } \\
R: \text { ATGCGTCCACAAAACCAATCTTCA }\end{array}$ & 61 \\
\hline${ }^{\mathrm{M}} \mathrm{P} 15 \mathrm{M} 51-204^{V}$ & $\begin{array}{l}F: \text { CGGAGGAAACATGGACAACGAA } \\
R: \text { AGCGAGCTCACTGCCAATCTACC }\end{array}$ & 56 \\
\hline${ }^{\mathrm{M}} \mathrm{P} 14 \mathrm{M} 54-252^{S}$ & $\begin{array}{l}F: \text { AGACCAGCATTACCTAAGCAGAGA } \\
R: \text { AGAGGAGAGTGAGTGTAGGTGTCG }\end{array}$ & 56 \\
\hline${ }^{\mathrm{M}}$ besV76P5D5AR ${ }^{V}$ & $\begin{array}{l}F: \text { GAGGAGCCGTGTCGTCTTGT } \\
R: \text { CCGTTTCCGTTCACTGGTTAT }\end{array}$ & 56 \\
\hline${ }^{\mathrm{M}}$ besS35P2K14EF ${ }^{S}$ & $\begin{array}{l}F: \text { TTGAAACAGCTGGGGTCTT } \\
R: \text { TGGTACACAAATATTCGTCTGC }\end{array}$ & 58 \\
\hline${ }^{\mathrm{MG}} R p h q 2 . \mathrm{S}_{01}{ }^{S}$ & $\begin{array}{l}F: \text { TGAAGGCGGGTTTGGTGTGGTGTA } \\
R: \text { CCCGCGTATGATTCTCTGCCTCTT }\end{array}$ & 58 \\
\hline${ }^{\mathrm{MG}} R p h q 2 . \mathrm{V} 30^{S}$ & $\begin{array}{l}F: \text { CGGCGGTGCGATCATAGAAT } \\
R: \text { TCCCCGGCCGTAGAGTCC }\end{array}$ & 65 \\
\hline${ }^{\mathrm{G}} R p h q 2 . \mathrm{V} 32^{S}$ & $\begin{array}{l}F: \text { GGGGCCCCGGCTATCGTGTA } \\
R: \text { AACTTTCCGCGGCAATCCTTCTTCT }\end{array}$ & 65 \\
\hline${ }^{\mathrm{a}} \mathrm{S} 35 \mathrm{P} 100001 \mathrm{~F} 4^{S}$ & $\begin{array}{l}F: \text { CCTCGCTAGTCAAGGAGGTG } \\
R: \text { GTGGCTGTTGTAGGGACGAT }\end{array}$ & 65 \\
\hline${ }^{\mathrm{a}} \mathrm{S} 35 \mathrm{P} 100004 \mathrm{~F} 2^{S}$ & $\begin{array}{l}F: \text { TTAATTTCTGCTCGCGTGTG } \\
R: \text { TGCATGCACTCCTCGTTTAG }\end{array}$ & 65 \\
\hline${ }^{\mathrm{M}} \mathrm{S} 7300002 \mathrm{~F}^{S}$ & $\begin{array}{l}F: \text { GACGTTGAGGAGAGCAAAGG } \\
R: \text { GCCGTTTATCACGAGGTTGT }\end{array}$ & 65 \\
\hline
\end{tabular}

$T_{\mathrm{a}}$ is the annealing temperature

м A molecular marker

G An annotated gene

MG An annotated gene converted into a marker

a A short DNA fragment at the edge of a gap in assembled BAC sequences

$S$ Primer pair used to screen only the BAC library of SusPtrit

${ }^{V}$ Primer pair used to screen only the BAC library of Vada

VS Primer pair used to screen the BAC library of Vada as well as SusPtrit
RiceGAAS (Sakata et al. 2002; http://ricegaas.dna.affrc. go.jp/). Since the RiceGAAS is dedicated to rice and not barley, there is a chance for false positives when applied to barley sequences. Therefore, the sequence of the predicted genes were used to blast against the barley expressed sequence tag (EST) tentative consensus (TC) sequences from the barley TIGR Gene Indices database (http:// www.tigr.org/tdb/tgi/index.shtml). A predicted gene was retained if the best blast hit was above a threshold e value $\leq 1.0 \mathrm{E}-15$. Comparison was made between the gene annotations by TriAnnot_v3.5 and RiceGAAS pipelines.

\section{Transcriptome sequencing}

The results from the annotation were compared with transcriptome data obtained from leaf tissue of L94 and Vada.
Tissue was collected from the first leaf of seedlings and from flag leaves of L94 and Vada at 0, 6, 12 and $48 \mathrm{~h}$ after inoculation with $P$. hordei. The samples were processed by the Beijing Genomics Institute (http://www.genomics.cn/en/index). The RNA isolation and cDNA synthesis were performed according to the TruSeq ${ }^{\mathrm{TM}}$ RNA sample preparation guide from Illumina (Catalog \# RS-930-20 01, Part \# 15008136 Rev. A, November 2010). After extraction, RNA samples were combined in aliquots to obtain two pools of leaf RNA, one for L94 and one for Vada. The cDNA libraries were constructed for each pool and sequenced on an Illumina HiSeq ${ }^{\mathrm{TM}}$ iSeqq. The software TopHat was used to map the raw reads of transcriptome data on the previously assembled BAC contig sequences from Vada and SusPtrit. The mapping results were used to improve the annotation of the predicted genes and to 
Table 2 Primer pairs for specific amplification of selected Vada- and SusPtritspecific genes

\begin{tabular}{lllll}
\hline Name & Gene & Line & Primers sequences $\left(5^{\prime}-3^{\prime}\right)$ & $T_{\mathrm{a}}\left({ }^{\circ} \mathrm{C}\right)^{\mathrm{a}}$ \\
\hline V.Perox-3 & Peroxidase & Vada & $F$ : CGTATGGGTTTGTAGGTGTAGCAT & 60.4 \\
& & & $R$ : CAGTTCGCCAAGTCGATGACCA & 62.8 \\
S.UF & \multirow{2}{*}{ Unknown function } & \multirow{2}{*}{ SusPtrit } & $F$ : CCGAGATCCTTGTTGCACTATTAC & 59.4 \\
& & & $R$ : GGTATACCTGTCACTAACAAACACT & 58.7 \\
S.Kin-1 & Kinase & \multirow{2}{*}{ SusPtrit } & $F$ : CCGGTACAGTCCATGTTTTCTC & 59.5 \\
& & & $R$ : CTCAGTGCTTCAGATGTTGCTTAG & 60.0 \\
\hline
\end{tabular}

${ }^{\mathrm{a}} T_{\mathrm{a}}\left({ }^{\circ} \mathrm{C}\right)$ annealing temperature obtain information on their expression in infected and noninfected leaf tissues.

\section{Marker saturation of the regions containing $R p h q 2$}

Between the flanking markers (WBE114 and WBE115), $\mathrm{BAC}$ end sequences and annotated gene sequences were used to develop sequence characterized amplified regions (SCAR) and cleaved amplified polymorphic sequence (CAPS) markers, polymorphic between Vada, SusPtrit and L94. Markers polymorphic between Vada and L94 were genetically mapped using the homozygous recombinant plants from Marcel et al. (2007a) to confirm their position and order. SCAR and CAPS markers were also developed from the AFLP fingerprints by converting polymorphic bands to single locus PCR markers following the strategy proposed by Brugmans et al. (2003). Primers were designed using Lasergene software (DNASTAR ${ }^{\circledR} 8$ Inc., Madison, WI, USA). The markers developed were used to assist in ordering the BAC clones.

WBE114 and WBE115 (Table 1) together with two newly developed markers, viz. Rphq2.S01 (Table 1) and Rphq2.V09; F: 5'-GCCTCTACTTCCACGACTGC-3', R: 5'-CCGGAGATGAC GATGATGT-3') were used to screen the Morex BAC library (Nils Stein, Leibniz Institute of Plant Genetic and Crop Plant Research, IPK) to identify the homologous Rphq2 region in the Morex genomic sequence.

\section{Haplotypes of one Vada-specific and two SusPtrit-specific genes in barley accessions}

A large part of the sequence bracketed by WBE114 and WBE115 had little or no homology between Vada and SusPtrit, and contained several cultivar-specific genes. We determined the occurrence of one Vada-specific (V.Perox-3) and two SusPtrit-specific genes (S.UF and S.Kin-1) in 194 barley accessions, including Vada and SusPtrit. These three genes were expressed in leaf tissue and might be considered candidate genes for the Rphq2 gene. Most (120) of the accessions had been used in an association study by Kraakman et al. (2006), but we added 74 accessions. Those additional accessions came from a panel of barley accessions previously used to study nonhost resistance (Atienza et al. 2004), or were used to generate mapping populations (like Golden Promise and Steptoe) or featured as important ancestors of European barley cultivars. Primer pairs with specific amplification for the Vada- and SusPtrit-specific genes were designed using DNAMAN (Table 2). A primer pair was designed to amplify part of an exon and part of an intron to ensure specific amplifications.

A haplotype of a barley accession is based on the presence (P) and absence (A) of V.Perox-3, S.UF and S.Kin-1. A haplotype of 'PAA' indicates the presence of V.Perox-3 and the absence of S.UF and S.Kin-1; 'APP' the absence of V.Perox-3 and the presence of S.UF and S.Kin-1.

\section{Comparative mapping in barley, rice and Brachypodium}

The sequence of annotated genes in the refined region containing Rphq2 was used for blast searches of rice and Brachypodium distachyon homologous genes, in the Rice Genome Annotation Project blast search (http://rice.plantbiology.msu.edu/analyses_search_blast.shtml) and in the B. distachyon blast portal (http://blast.brachypodium.org/), respectively. For each annotated gene in barley, the best blast hit was retained above a threshold $e$ value $\leq 1.0 \mathrm{E}-15$.

\section{Results}

\section{Construction and characterization of the BAC libraries}

The Vada library was organized in 116 pools named V1 to V116 (Supplemental Table 1), containing an average of 1435 clones per pool. Based on the count of blue (non-recombinant) and white (recombinant) colonies per plate, the percentage of recombinant clones was estimated to be $96.8 \%$. Thus, the library consists of approximately 161,000 recombinant clones. The average size of inserts ranges from 67 to $98 \mathrm{Kbp}$. The observed insert sizes for each fraction do not correspond well to the expectations based on the size selection (Supplemental Table 2). 
The SusPtrit library was organized in 110 pools named S1 to S110 (Supplemental Table 3), containing an average of 1606 clones per pool. The percentage of recombinant clones was estimated to be $97.9 \%$ based on the count of blue and white colonies per plate. Thus, the library consists of approximately 173,000 recombinants clones. The average size of SusPtrit inserts ranged from $107 \mathrm{Kbp}$ for selected fraction $\mathrm{H} 1-141 \mathrm{Kbp}$ for selected fraction H3 (Supplemental Table 4).

Based on a haploid barley genome size of $4.98 \mathrm{~Gb}$ (The International Barley Genome Sequencing Consortium 2012) and on the genome coverage of each fraction of the libraries (Supplemental Tables 2 and 4), we estimated that the coverage of the Vada and SusPtrit BAC libraries are approximately 2.6 and 3.7 genome-equivalents, respectively. Together, the libraries cover 6.4 genome-equivalents that allow for a probability greater than $99 \%$ of recovering any specific sequence from the barley genome (Clarke and Carbon 1976).

To verify the genome representation of the libraries, we screened 46 pools of Vada (V1-V46) and 46 pools of SusPtrit (S1 to S46) corresponding approximately to 1.4 and 1.9 genome-equivalents, respectively, with 14 microsatellite markers (Supplemental Table 5). An average of 2.7 positive pools per microsatellite marker was obtained for the Vada library and an average of 3.5 positive pools for the SusPtrit library (Supplemental Table 5). All markers were represented at least once in the 46 pools of the SusPtrit library and only two markers were not represented in the 46 pools of the Vada library, indicating that the overall barley genome is well represented in our BAC libraries. Based on the average representation of the 14 microsatellite markers in 46 pools per library, we estimated that the total coverage of the Vada and SusPtrit BAC libraries are 5.0 and 6.8 genome-equivalents, respectively.

\section{Generation of Vada and SusPtrit sequences at Rphq2}

We used four primer pairs for the BAC library of Vada and ten primer pairs for the BAC library of SusPtrit (Table 1) to screen for BAC clones spanning the Vada or SusPtrit allele at $R p h q 2$. For Vada, the four primer pairs detected 16 positive BAC pools in total from the Vada BAC library and for SusPtrit the ten primer pairs detected a total of 21 positive BAC pools in the SusPtrit BAC library (Supplemental Table 6). The BAC pools positive for two or more primer pairs were prioritized for the identification of single positive BAC clones. Finally, we validated seven Vada BAC clones originating from four Vada BAC pools and 17 SusPtrit $\mathrm{BAC}$ clones originating from nine SusPtrit BAC pools. All BAC clones were fingerprinted. The order of the BAC clones was not only based on the BAC fingerprint, but also on the primer amplification (Supplemental Table 7).

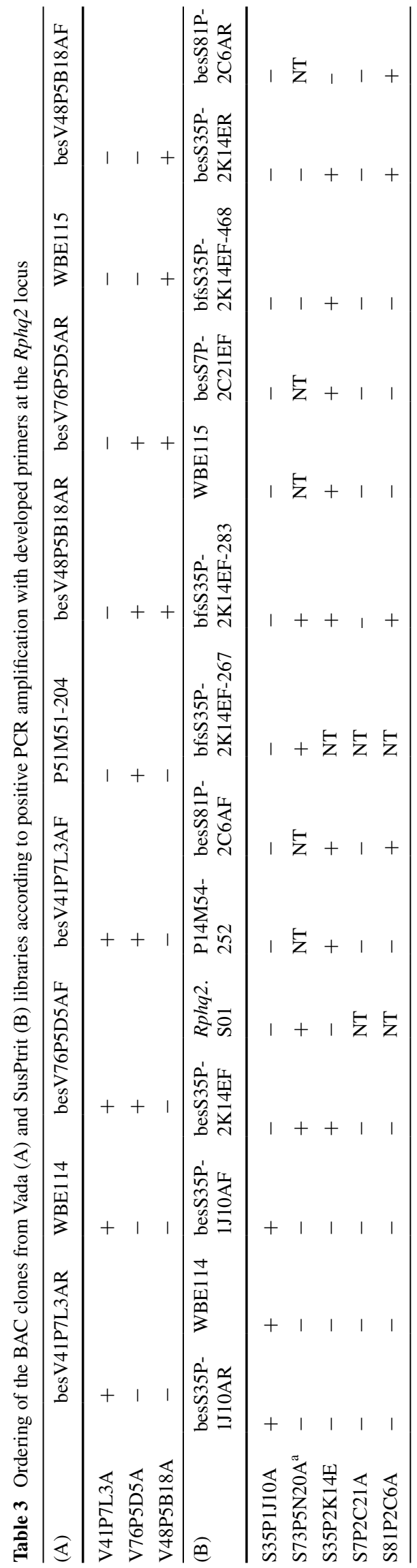


From the seven BAC clones of Vada, the AFLP fingerprint revealed a minimum tiling path of only three clones [V41P7L3A (120 Kbp), V76P5D5A (87 Kbp) and V48P5B18A (150 Kbp)], which were overlapping each other to cover the Rphq2 genetic window between markers WBE114 and WBE115 (Table 3). Those three BAC clones were sequenced following both Sanger and 454 sequencing approaches. The sequences obtained with one or the other approaches were assembled independently. The sequence assembly obtained from Sanger sequencing had ten gaps between WBE114 and WBE115, while the sequence assembly obtained from 454 sequencing had 12 gaps. Aligning both assemblies led to a consensus sequence with only two gaps left, one in the sequence of V76P5D5A and the other one in the sequence of V48P5B18A. Together, the two gaps represent approximately $7 \mathrm{Kbp}$, calculated by comparing the estimated insert size of each BAC clone with the size of the consensus sequence. The physical length of the three contigs covering the Vada BAC sequence from WBE114 to WBE115 is approximately $195 \mathrm{~kb}$ excluding the gaps.

From the 17 BAC clones of SusPtrit, six had an identical AFLP fingerprint with S35P1J10A (105 Kbp) which harbored WBE114, but were not overlapping with any of the other identified SusPtrit BAC clones. On the other side, three BAC clones positive for WBE115 were overlapping with each other [S35P2K14E (140 Kbp), S7P2C21A $(80 \mathrm{Kpb})$ and S81P2C6A (135 Kbp)], and with a fourth BAC clone [S73P5N20A (165 Kbp)] (Table 3). S35P2K14E was selected for sequencing because it was positive not only for WBE115 but also for P14M54-252, a marker mapped closer to Rphq2 (Marcel et al. 2007a; Fig. 1). S73P5N20A was also selected for sequencing because it overlapped with S35P2K14E distal to WBE115, and was expected to extend further into the Rphq2 genetic window. The BAC clone S35P2K14E was sequenced following both Sanger and 454 sequencing approaches while the other two BAC clones S73P5N20A and S35P1J10A were sequenced following a 454 sequencing approach only. The sequences obtained with one or the other approaches were assembled independently. The sequence assembly of S35P2K14E from Sanger sequencing had six gaps, while the sequence assembly obtained from 454 sequencing for this clone had four gaps. Aligning both assemblies led to a consensus sequence of $122 \mathrm{Kbp}$ with no gap for S35P2K14E. The sequence assemblies for the other two SusPtrit clones were composed of four contigs each (three gaps for each clone). The consensus sequence of the overlapping BAC clones S73P5N20A and S35P2K14E resulted in three contigs of sizes $139 \mathrm{Kbp}, 563 \mathrm{bp}$, and $60 \mathrm{Kbp}$. The length of the available consensus SusPtrit sequence between WBE114 and WBE115 is $226 \mathrm{Kbp}$. But the SusPtrit DNA stretch going from WBE114 to WBE115 shall be slightly longer as the SusPtrit contig was not entirely covered with overlapping BAC clones.

\section{Sequence annotation}

The pipeline TriAnnot_v3.5 (Leroy et al. 2012) predicted 12 genes on both Vada and SusPtrit contigs. The RiceGAAS pipeline predicted 33 genes for the Vada contig and 50 for the SusPtrit contig. Among the 33 predicted genes for Vada, 22 hit a barley EST in TIGR Gene Indices database above a threshold $e$ value $\leq 1.0 \mathrm{E}-15$. Among the 50 predicted genes for SusPtrit, 34 had a hit above the same threshold. In Vada as well as in SusPtrit 11 genes were common to the predictions of both TriAnnot_v3.5 and RiceGAAS pipelines.

The pipeline TriAnnot_v3.5 provided a more reliable prediction due to its specificity and sensitivity compared to RiceGAAS (Leroy et al. 2012). This is because RiceGAAS depends solely on ab initio predictions and was trained for annotating rice sequences rather than barley sequences. The pipeline TriAnnot_v3.5 predicts genes by combining ab initio prediction and biological evidence based on sequence similarity with known coding sequences (transcripts/proteins) from grass genomes, and the specific barley default setting was used for the analysis (version v3.5). Consequently, we present in detail the genes predicted for the Vada and SusPtrit contigs using TriAnnot_v3.5. For the Vada contig, the predicted genes encoded three peroxidases (V.Perox-1, -2, -3), two kinases (V.Kin-1, -2), one seven-in-absentia protein family member (V.SINA), one protein with a legume lectin domain (V.Leg-Lec-D), and five proteins with unknown function/or hypothetical/or uncharacterized (V.UF/HP/ UP). Among the twelve predicted genes from the Vada sequence, V.Perox-1, -2, -3 and V.Kin-1 were supported by sequences from the transcriptome data of Vada, indicating their expression in leaf tissue. For the SusPtrit contig, the predicted genes encoded two peroxidases (S.Perox-1, -2), three kinases (S.Kin-1, -2, -3), and seven proteins with unknown function or hypothetical or uncharacterized (S.UF/HP/UP). Among the twelve predicted genes from SusPtrit sequence, S.Perox-1, -2, S.UF and S.Kin-1 and -2 were supported by sequences from transcriptome data of L94. The comparison of genes predicted in both cultivars indicated that V.Perox-1, V.Perox-2, V.UP, V.Kin-1 and V.Kin-2 from Vada were positioned at the same locus (allelic) as S.Perox-1, S.Perox-2, S.UP, S.Kin-2 and S.Kin-3 from SusPtrit, respectively. Their positions were supported by co-dominant markers (Fig. 1). The common genes between Vada and SusPtrit shared at least $99 \%$ sequence similarity except for V.Kin-1 and S.Kin-2 which 
(A)

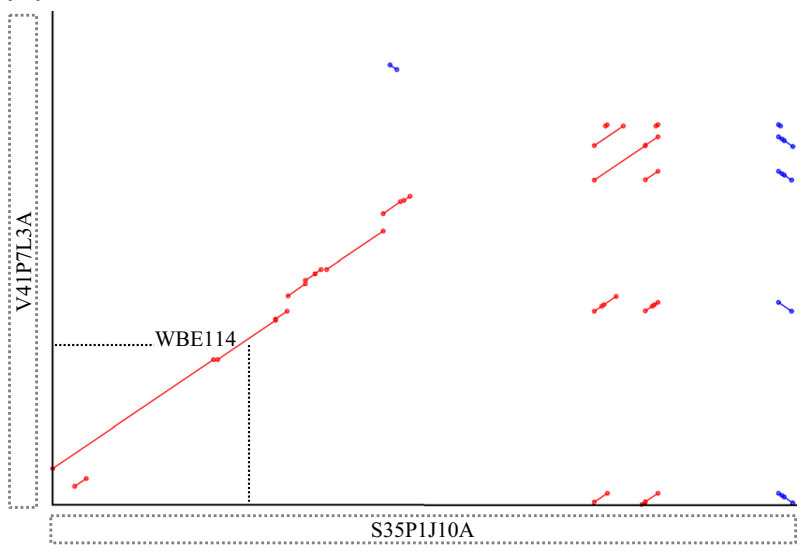

Fig. 2 Dot plots suggesting lack of homology between the sequences of Vada and SusPtrit at Rphq2. Red dots indicate the sequence similarity between Vada and SusPtrit with the same orientation. Blue dots indicate the sequence similarity but either the Vada or SusPtrit

Table 4 Alignment of 18 Morex sequence contigs on Vada and SusPtrit sequence assemblies between WBE114 and WBE115 (corresponding to Morex BAC contig 44195)

\begin{tabular}{|c|c|c|}
\hline Morex contigs; (sequence size, bp) & $\begin{array}{l}\text { Align to } \\
\text { Vada }\end{array}$ & $\begin{array}{l}\text { Align to } \\
\text { SusPtrit }\end{array}$ \\
\hline Mor_cont 43090; (5993)/WBE114 & Yes $^{\mathrm{b}}$ & Yes \\
\hline Mor_cont 53633; (3569) & Yes & No \\
\hline Mor_cont 2267159; (240) & No & Yes \\
\hline Mor_cont 88428; (1710) & No & Yes \\
\hline Mor_cont 2550490; (9192) & No & Yes \\
\hline Mor_cont 2343918; (327) & No & Yes \\
\hline Mor_cont 41082; (7623) & No & Yes \\
\hline Mor_cont 442275; (1129) & No & Yes \\
\hline Mor_cont 280043; (1061) & No & Yes \\
\hline Mor_cont 1590687; (2251) & Yes & Yes \\
\hline Mor_cont 287733; (1989) & Yes & Yes \\
\hline Mor_cont 224400; (1060) & Yes & Yes \\
\hline Mor_cont 321571; (308) & Yes & Yes \\
\hline Mor_cont 60124; (3413) & Yes & Yes \\
\hline Mor_cont 1588307; (1769) & Yes & Yes \\
\hline Mor_cont 1572547; (2594) & Yes & Yes \\
\hline Mor_cont 8886; (1904) & Yes & Yes \\
\hline Mor_cont $2546833 ;(3163) / \mathrm{WBE} 115^{\mathrm{c}}$ & Yes & Yes \\
\hline
\end{tabular}

a WBE114 is 301 bp distal from Mor_cont 43090

${ }^{b}$ Yes means that the Morex sequence aligned to the subject with a minimum sequence match of $80 \%$

c The WBE115 sequence of Vada aligned to Mor_cont 2546833 with one single nucleotide polymorphism and two for the WBE115 sequence of SusPtrit

had only $90 \%$ sequence similarity. The other annotated genes were not shared between Vada and SusPtrit (Fig. 1). The V.UF and V.HP from Vada did not align to the S.UF
(B)

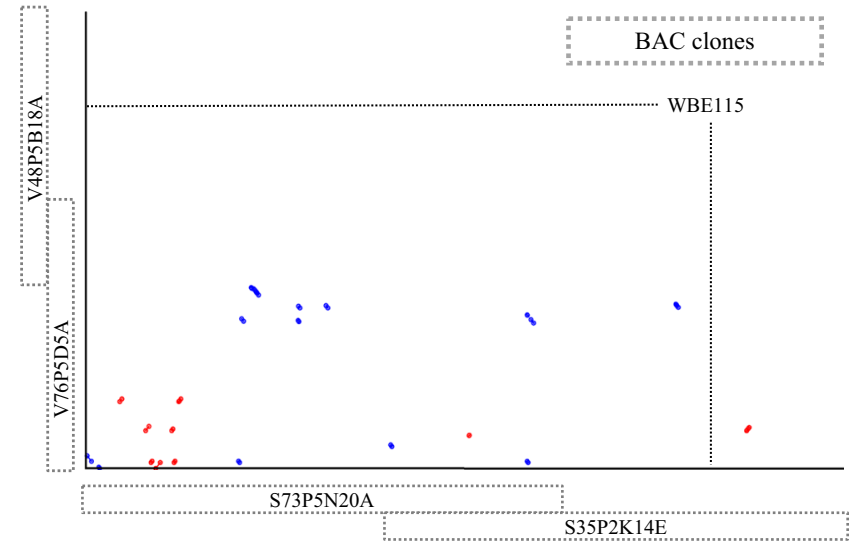

sequence is inverted. a Alignment between the sequences of Vada and SusPtrit BAC clones proximal to the gap in SusPtrit BAC contig. b Alignment between the sequences of Vada and SusPtrit BAC clones distal to the gap in SusPtrit BAC contig (color figure online)

and S.HP from SusPtrit, respectively, and were regarded as different loci. The RiceGAAS pipeline predicted one additional kinase in SusPtrit. That sequence hit a barley EST in TIGR Gene Indices database with an $e$ value of 1.0E-34. The likelihood of genes listed in Fig. 1 to be candidates for $R p h q 2$ will be discussed in the "Discussion".

Using the sequence information between WBE114 and WBE115, we developed 39 new markers that were polymorphic between Vada and SusPtrit, and also determined their alleles for the line L94 carrying the susceptibility allele at Rphq2. Interestingly, SusPtrit and L94 have for all those markers the same allele. Together with two AFLPconverted single locus PCR markers developed by Marcel et al. (2007a), 41 markers were mapped between the flanking markers WBE114 and WBE115 (Supplemental Table 8). Among the 41 markers, 20 were dominant markers amplifying only Vada DNA and 17 were dominant markers amplifying only SusPtrit DNA (Fig. 1). Since the markers were developed by amplifying whole genome DNA, the dominance of the markers indicates that the sequences were Vada- and SusPtrit specific, respectively. There were four co-dominant markers; three were developed based on the common annotated genes between Vada and SusPtrit and one based on a random sequence from one of the sequence contigs of S73P5N20A. According to the sequence annotation and to the dominant nature of most markers located between WBE114 and WBE115, there seems to be a lack of homology between Vada and SusPtrit for the region containing Rphq2. This is further supported by a dot plot analysis (MUMmer) which compares the sequence of Vada and SusPtrit between WBE114 and WBE115 (Fig. 2). Sequence similarity is only observed on approximately the first $36 \mathrm{Kbp}$ of S35P1J10A which includes WBE114. 


\section{Homology of the region with other barley germplasm}

We searched in the Morex genomic sequence (The International Barley Genome Sequencing Consortium 2012) the sequence corresponding to the divergent $R p h q 2$ region between Vada and SusPtrit. Two pairs of primers, respectively, amplifying the predicted genes V.Perox-2 (Rphq2. V09) and S.Kin-1 (Rphq2.S01), detected five overlapping BAC clones from Morex covering the Rphq2 region (HVVMRXALLEA0011E05, HVVMRXALLEA0179K22, HVVMRXALLeA 0269J17, HVVMRXALLeA0278A17, and HVVMRXALLeA0299N24). The BAC clones are positioned between 806 and $1101 \mathrm{~kb}$ of contig $44195(1.9 \mathrm{Mb})$ on chromosome 2HL (http://mips. helmholtz-muenchen.de/plant/barley/index.jsp). A total of 118 Morex sequence contigs (Mor_cont) from the BAC contig 44195 (kindly provided by Nils Stein, Leibniz Institute of Plant Genetic and Crop Plant Research, IPK) were aligned to the sequence between WBE114 and WBE115 of Vada and SusPtrit using Lasergene software with a minimum sequence match of $80 \%$ (DNASTAR ${ }^{\circledR} 8$ Inc., Madison, WI, USA). Eleven of the 118 Mor_cont from BAC contig 44195 aligned to the Vada sequences and 17 to the SusPtrit sequences (Table 4). We found that Mor_cont 43090 was approximately 300 bp distal from WBE114, and that Mor_cont 2546833 aligned to a region including WBE115. We estimated the physical distance between WBE114 and WBE115 of Morex to be of approximately $254 \mathrm{~kb}$, which is $28 \mathrm{~kb}$ longer than the estimated physical distance in SusPtrit. More sequences from SusPtrit than from Vada could be aligned to the Morex sequences, indicating that SusPtrit and Morex are likely to be more similar than Vada and Morex at the Rphq2 locus.

We also developed specific primers to amplify V.Perox-3 (Vada specific), S.UF and S.Kin-1 (SusPtrit specific) to determine whether these genes were present in 194 barley accessions. Based on the presence (P) and absence (A) of the three genes, all the barley accessions can be grouped into two about equally common haplotypes viz., PAA (111 accessions, $57 \%$, including Vada and $H$. laevigatum) and APP (83 accessions, $43 \%$, including SusPtrit, Morex and L94) (Supplemental Table 9). We should note that "presence" of a gene does not imply that the accessions have identical sequences for the gene, and that "absence" refers to the lack of amplification. An accession for which a gene is scored as "absent" may have some version of the gene, but with too much sequence difference for the primers to amplify the DNA.

\section{The synteny between barley, rice and Brachypodium}

The Rphq2 region is located in the barley $2 \mathrm{~L} 1.0$ region, which has a syntenic relationship with a region on rice
Chromosome 4 (Marcel et al. 2007a) and a region on Brachypodium Chromosome 5 (Mayer et al. 2011). Homologs of the two genes used to develop markers WBE114 and WBE115 have indeed been identified on rice Chromosome 4 and on Brachypodium Chromosome 5 (Table 5). The interval between the rice homologs Loc_Os04g59260 and Loc_Os04g59320 contains five annotated genes encoding a hypothetical protein, two retrotransposon proteins, a strictosidine synthase, and a phospholipase $\mathrm{C}$. The interval between the Brachypodium homologs Bradi5g27210 and Bradi5g27240 contains 2 genes encoding a peroxidase and a phospholipase $\mathrm{C}$. The gene used to develop the marker WBE114 encodes a peroxidase precursor. Interestingly, this gene is present in a single copy in rice, in two copies in Brachypodium and in barley line SusPtrit, and in three copies in barley cultivar Vada (Table 5). In addition, only two additional genes seem to be shared between the three species and the two barley genotypes, i.e., a phospholipase $\mathrm{C}$ and a protein kinase used to develop marker WBE115. The sequence of the phospholipase $\mathrm{C}$ genes in rice and Brachypodium are highly homologous to the genes encoding the uncharacterized proteins V.UP and S.UP (Table 5). Therefore, V.UP and S.UP may correspond to phospholipase $\mathrm{C}$ genes. The remaining seven genes predicted only from Vada and seven genes predicted only from SusPtrit have no homolog in the identified synthetic regions of rice and Brachypodium. Nevertheless, some of those genes had a blast hit with value $\leq 1.0 \mathrm{E}-15$ with rice or Brachypodium genes located outside of the Rphq2 syntenic interval (Table 5).

\section{Discussion}

\section{Two barley BAC libraries for the isolation of genes involved in basal resistance}

Two non-gridded BAC libraries were constructed for Vada and SusPtrit barley genotypes. The average insert size over the complete Vada BAC library was $81 \mathrm{Kbp}$, with individual clones ranging from 18 to $209 \mathrm{Kbp}$ (Supplemental Fig. 2a; Supplemental Table 2). The average insert size over the complete SusPtrit BAC library was $108 \mathrm{Kbp}$, with individual clones ranging from 33 to $274 \mathrm{Kbp}$ (Supplemental Fig. 2b; Supplemental Table 4). This variation in insert sizes corresponds to the size selected fractions of DNA fragments ranging from 50 to $250 \mathrm{Kbp}$ (i.e., fractions $\mathrm{H} 1-\mathrm{H} 3)$. On average, SusPtrit inserts are $27 \mathrm{Kbp}$ longer than Vada inserts. The average insert size of the Vada BAC library is the smallest among the BAC libraries available for barley. Such small average insert size is also observed in BAC libraries from other plant species such as wheat (Janda et al. 2004; Nilmalgoda et al. 2003) and 
Table 5 Best blast hits $(\leq 1.0 \mathrm{E}-15)$ of the predicted genes in Vada and SusPtrit sequences with the rice and the Brachypodium gene catalogs

\begin{tabular}{|c|c|c|c|c|c|}
\hline Vada & Rice & Brachypodium & SusPtrit & Rice & Brachypodium \\
\hline V.Perox- $1^{\mathrm{a}}$ & Loc_Os04g59260 (5.6E-127) & Bradi5g27210 (1.3E-134) & S.Perox- $1^{\mathrm{a}}$ & Loc_Os04g59260 (3.4E-128) & Bradi5g27210 (2.7E-134) \\
\hline $\mathrm{Na}$ & & & S.HP-1 & $\mathrm{x}$ & $\mathrm{x}$ \\
\hline V.Perox-2 & Loc_Os04g59260 (3.9E-103) & Bradi5g27220 (9.7E-119) & S.Perox-2 & Loc_Os04g59260 (1.0E-103) & Bradi5g27220 (3.0E-119) \\
\hline $\mathrm{Na}$ & & & S.HP-2 & $\mathrm{x}$ & $\mathrm{x}$ \\
\hline $\mathrm{Na}$ & & & S.HP-3 & $\mathrm{x}$ & $\mathrm{x}$ \\
\hline $\mathrm{Na}$ & & & S.UF & LOC_Os07g35310 (4.2E-61) & Bradi1g25552 (3.0E-73) \\
\hline V.HP-1 & $\mathrm{x}$ & $\mathrm{x}$ & $\mathrm{Na}$ & & \\
\hline V.SINA & LOC_Os05g06070 (1.7E-32) & Bradi2g01770 (7.7E-43) & $\mathrm{Na}$ & & \\
\hline V.Perox-3 & Loc_Os04g59260 (3.8E-108) & Bradi5g27220 (4.1E-120) & $\mathrm{Na}$ & & \\
\hline V.Leg-Lec-D & LOC_Os08g03002 (4.4E-60) & Bradi1g28320 (7.2E-63) & $\mathrm{Na}$ & & \\
\hline $\mathrm{Na}$ & & & S.HP-4 & LOC_Os11g30140 (1.7E-24) & $\mathrm{x}$ \\
\hline $\mathrm{Na}$ & & & S.HP-5 & $\mathrm{x}$ & $\mathrm{x}$ \\
\hline V.HP-2 & $\mathrm{x}$ & $\mathrm{x}$ & $\mathrm{Na}$ & & \\
\hline V.UF-1 & LOC_Os10g37260 (1.4E-66) & Bradi3g30820 (4.4E-63) & $\mathrm{Na}$ & & \\
\hline V.UF-2 & LOC_Os10g37260 (4.0E-28) & Bradi3g30820 (1.2E-20) & $\mathrm{Na}$ & & \\
\hline $\mathrm{Na}$ & & & S.Kin-1 & LOC_Os07g35310 (1.4E-136) & Bradi1g25552 (5.0E-194) \\
\hline V.Kin-1 & LOC_Os08g03020 (5.4E-140) & Bradi1g28320 (8.7E-225) & S.Kin-2 & LOC_Os08g02996 (4.6E-145) & Bradi1g28320 (3.5E-221) \\
\hline V.UP & Loc_Os04g59310 (5.3E-138) & Bradi5g27230 (7.0E-160) & S.UP & Loc_Os04g59310 (2.8E-137) & Bradi5g27230 (1.9E-159) \\
\hline V.Kin- $2^{\text {b }}$ & Loc_Os04g59320(1.5E-201) & Bradi5g27240 (2.4E-222) & S.Kin- $3^{b}$ & Loc_Os04g59320(1.5E-201) & Bradi5g27240 (2.4E-222) \\
\hline
\end{tabular}

For abbreviations see the footnotes under Fig. 1

Italic boxes indicate the syntenic interval of Rphq2 with rice chromosome 4 (indicated by Loc_Os $\underline{\mathbf{0 4}}$ ) and Brachypodium chromosome 5 (indicated by Bradi $\underline{\mathbf{5}}$ )

$\mathrm{Na}$ not annotated, $x$ no hit or no significant hit

a WBE114 is located within this annotated gene

b WBE115 is located within this annotated gene

soy bean (Xia et al. 2014). The average insert size of the Vada and SusPtrit BAC libraries is still comparable to three of the Morex BAC libraries (HVVMRXALLrA, HVVMRXALLhB and HVVMRXALLhC) recently made available by Schulte et al. (2011). However, the average insert size of the constructed BAC libraries is smaller than the one reported for the Cebada Capa BAC library (140 Kbp) which was constructed using the same protocol (Isidore et al. 2005). If a BAC library is to be used for genome-wide physical mapping and genome sequencing, then maximizing the average size of inserts is essential to limit walking. However, if a BAC library is to be used for positional cloning of genes that have already been confined to a very small interval, then having a large number of clones is more important to increase the chance to find the gene of interest. The principal aim of our BAC libraries is to isolate genes involved in basal resistance to cereal rust fungi after their high-resolution genetic mapping.

Based on the observed insert sizes of the BAC clones, the estimated genome coverage of the Vada BAC library is $2.6 \times$ and of the SusPtrit BAC library 3.7 $\times$. The genome coverage of the Vada library is comparable to the genome coverage of the Morex HVVMRXALLhB library, and
SusPtrit to HVVMRXALLeA (Schulte et al. 2011). The estimation of the genome coverage based on microsatellite markers indicates, however, coverage of $5.0 \times$ and $6.8 \times$ for the Vada and the SusPtrit BAC libraries, respectively. The discrepancy between both estimations may be due to an underestimation of the average size of the BAC clones. As it is often observed in monocots (Peterson et al. 2000), several bands of identical sizes may be obtained after NotI restriction of BAC clones, which may result in the underestimation of the insert size from some clones. On the other hand, half of the microsatellite markers used for screening the BAC pools were derived from barley ESTs/genes (i.e., EST-SSR markers), implying that a marker amplifying a member from a gene family may in some cases amplify other genes from the same family as well unless the sequences of the primer pairs are unique (Thiel et al. 2003). Indeed, the pressure of a primer to anneal on a similar but not identical sequence is much stronger on BAC DNA than it is on full genomic DNA. Therefore, the genome coverage of the two libraries remains uncertain, but is probably slightly higher for SusPtrit than for Vada. Based on insert sizes, the BAC library of Vada gives at least $93 \%$ probability of identifying a clone corresponding to any sequence of 
Vada and for BAC library of SusPtrit a probability of $98 \%$ is expected (Clarke and Carbon 1976). Together, the two BAC libraries give more than $99 \%$ probability of recovering any specific sequence from the barley genome.

\section{Haplotype divergence at the Rphq2 partial resistance locus}

The Rphq2 locus of Vada was donated by H. laevigatum (Arru et al. 2003; Giese et al. 1993). The name of the latter accession is taxonomically invalid, since it suggests a different (wild) species in the Hordeum genus. However, it is perfectly crossable with $H$. vulgare accessions and also has the $H$. vulgare general morphology, including non-shattering spikes. Therefore, it should be regarded as $H$. vulgare. It occurs in the ancestry of many West-European cultivars, including Emir, Delta and Minerva [Hickey et al. 2012; Germplasm Resources Information Network (GRIN) http://www.ars-grin. gov/npgs/holdings.html] and their derivatives. SusPtrit was bred from a double cross; Menelik/L100//Trigo Biasa/Nigrinudum (GRIN; Atienza et al. 2004). The very low degree (or even absence) of homology in the Rphq2 region was supposed to be due to the West-European cultivar Vada and hence due to the donor line $H$. laevigatum that contributed this chromosome section (Marcel et al. 2007a). Other barley lines, viz the Ethiopian L94, the American cultivar Morex and SusPtrit as a descendant from various exotic barley accessions, seem to have maintained their homology in this area, as it appears from the alignment of the Morex genome sequences with the SusPtrit sequences and not with the Vada sequences (Table 4). Accordingly, the primer pairs designed on SusPtrit sequences did amplify DNA of L94 while primer pairs designed on Vada sequences did not. This lack of homology implies that having a BAC library from the parent donor of the resistance allele (Vada) is a necessity, and that if we would have relied only on the BAC library of Morex, we might have had great difficulties to acquire sequence information for Vada in that region and to identify the candidate genes for resistance.

Highly divergent sequences are usually implicated in suppression of recombination, as it was reported to occur commonly throughout the maize genome (Fu and Dooner 2002; Gore et al. 2009). Intraspecific haplotype divergence seems particularly common at resistance loci giving evidence for the dynamic evolution of those genome regions. Such haplotype divergence may arise from different mechanisms, including separate retrotransposon invasion between different genotypes of a same species $(R p h 7$ resistance to leaf rust in barley; Scherrer et al. 2005), gene duplication and unequal recombination in clustered multigene families (RPP5 resistance to Hyaloperonospora arabidopsidis in Arabidopsis: Noël et al. 1999) or introgression of new resistance/susceptibility genes from landraces or wild-related species into modern cultivars ( $Y r 36$ resistance to stripe rust in wheat: Fu et al.
2009; Tsn1 resistance to tan spot disease of wheat: Faris et al. 2013). Such divergent sequences have been systemically associated with suppression of recombination in the target regions, hampering map-based cloning of the genes of interest. Recently, the genetic dissection of the $S r 2$ adult plant stem rust resistance locus, introgressed from tetraploid emmer wheat into the modern hexaploid bread wheat cultivar Hope, revealed a major haplotype divergence including an insertion of a Germin-Like Protein (GLP) gene cluster in Hope (Mago et al. 2014). The GLP cluster represents a common presence-absence polymorphism in wheat but presence of the cluster does not correlate with the presence of $\mathrm{Sr} 2$, indicating that a GLP member of the cluster and not the cluster itself likely confers $\mathrm{Sr} 2$ resistance. Suppressed recombination due to strong haplotype divergence and presence of a complex cluster of GLPs render the map-based cloning of $\mathrm{Sr} 2$ a strenuous exercise (Mago et al. 2014).

Thus, a practical consequence of the strong haplotype divergence at the Rphq2 locus is that we do not expect further recombination between V.Perox-2/S.Perox-2 and V.Kin-1/S.Kin-2, as in heterozygous material this chromosome region will probably hardly pair. Indeed we failed to obtain recombinant plants in this region after screening more than 3000 plants (data not shown). The lack of recombination is also suggested by the repulsion between V.Perox-3 (Vada specific) and S.UF + S.Kin-1 (SusPtrit specific) in a set of 194 barley accessions, including some Ethiopian, Indonesian, East-Asian, American, European and $H$. spontaneum accessions. We labeled these haplotypes PAA (presence, absence absence) when they only amplified the V.Perox-3 sequence, and APP when they only amplified the S.UF and S.Kin-1 sequences. Among the 194 tested barley accessions, no other haplotype than the PAA and APP haplotypes were detected, suggesting an early and complete split in the haplotypes and a strict repression of recombination in this chromosome region. Suppression of recombination prevents further fine mapping of $R p h q 2$ using the Vada and SusPtrit or Vada and L94 heterozygous material. Nevertheless, among the 111 accessions having the PAA haplotype, we observed variation from a high level of partial resistance (including Vada and H. laevigatum) to a high level of susceptibility to barley leaf rust (data not shown). Susceptible PAA accessions are likely to carry a susceptible $r p h q 2$ allele and to recombine with the $R p h q 2$ locus in Vada, allowing further fine mapping of $R p h q 2$.

\section{Multiple candidate genes at the Rphq2 locus}

The annotation of sequences at Rphq2 using TriAnnot_v3.5 with the specific barley default analysis identified 12 genes in Vada and 12 genes in SusPtrit, but only five of the annotated genes are shared between Vada and SusPtrit. Out of the five annotated genes in common between Vada and SusPtrit, 
four were also conserved in rice and B. distachyon. No nucleotide-binding site-leucine-rich repeat (NBS-LRR) resistance gene was annotated in the Rphq2 region. A study of differentially expressed genes between L94 and L94-Rphq2 NIL suggested seven candidates for Rphq2 (Chen et al. 2010). Among the candidates, only one gene, unigene2111 (encoding a peroxidase), was similar to an annotated gene at $R p h q 2$. Unigene 2111 has $99 \%$ identity with the coding sequence of V.Perox-2 of Vada and S.Perox-2 of SusPtrit. This suggests peroxidase-2 as a good candidate to explain $R p h q 2$. This is also supported by the fact that peroxidases are known to be involved in defense reactions, i.e., cell wall reinforcement and hypersensitive reaction [reviewed in Hückelhoven and Kogel (2003); Almagro et al. (2009)]. Furthermore, González et al. (2010) found $61 \%$ of the QTLs for partial resistance to adapted rust fungi (including Rphq2) to co-localize with peroxidase-based markers. The kinases are also good candidates to explain $R p h q 2$. Kinases are involved in various signaling pathways including plant defense system against pathogens [Reviewed in Rodriguez et al. (2010); Antolín-Llovera et al. (2012)]. The annotated peroxidase and kinase genes co-segregating with $R p h q 2$ resistance phenotype have all been found to be expressed in leaf tissue after leaf rust infection, making them good candidate genes to explain Rphq2. It is possible that one of the peroxidase or kinase genes identified in the physical window of $R p h q 2$ affects the resistance phenotype observed. Another possibility is that peroxidase or kinase gene members function as a complex QTL as observed in rice where the resistance effect of a QTL on chromosome 8 was shown to be contributed by a cluster of germin-like protein genes (Manosalva et al. 2009).

A gene from the SINA protein family was annotated only on the Rphq2 sequence of Vada. Based on its function, this might be a candidate gene for $R p h q 2$ as well. SINA proteins are E3 ligases with a RING finger domain at the N-terminal followed by a conserved SINA domain which has a function in substrate binding and dimerization (Hu and Fearon 1999). One particular SINA protein is found, in a symbiotic interaction, to impair the rhizobial infection in Medicago truncatula (Mbengue et al. 2010) and Lotus japonicus (Den Herder et al. 2012). However, no support of expression was found for V-SINA in our RNA-seq data, making it a less likely candidate gene to explain $R p h q 2$. The candidate genes for $R p h q 2$ resemble none of the partial resistance genes cloned previously ( $\mathrm{Fu}$ et al. 2009; Fukuoka et al. 2009; Hayashi et al. 2010; Krattinger et al. 2009; Manosalva et al. 2009). Genes for partial resistance can be resistance factors, such as ABC transporter gene of Lr34 (Krattinger et al. 2009), or susceptibility factors, such as a proline-rich protein of Pi21 (Fukuoka et al. 2009). Therefore, the candidate genes of Rphq 2 might be either a resistance factor from Vada or a susceptibility factor from SusPtrit.

Stable transformation of candidate genes for resistance in a susceptible barley genotype can be performed using
Golden SusPtrit, a new genetically well-transformable barley line (Yeo et al. 2014). Golden SusPtrit inherited the susceptibility of SusPtrit to $P$. hordei and to non-adapted rust fungi, as well as the transformability of Golden Promise. The transformants in the genetic background of Golden SusPtrit will allow the testing of Rphq2 candidate genes affecting resistance to adapted and non-adapted rust fungi. This will be valuable information to understand partial resistance in barley and its possible association with non-host resistance.

Author contribution statement FYKS: did much of the experimental work; wrote the first draft, and implemented all suggestions by co-authors, YW: advised on the annotation and did the haplotyping of 194 accessions. TV: developed molecular markers. $\mathrm{CH}$ : assisted TCM in developing the BAC libraries. PL: did the sequences annotation using Triannot pipeline. BC: supervised TCM in developing the BAC library. XQQ: supervised the transcriptomics and annotation studies. REN: overall supervision, and editing of the various drafts and submission. TCM: designed the study, developed the BAC libraries, and actively supervised FYKS in writing.

Acknowledgments We particularly wish to thank Dr D. Schulte and Dr R. Arriyadasa (IPK, Gatersleben, Germany) for providing information and assistance concerning the exploration of the Morex BAC libraries. We are also grateful to Dr N. Stein (IPK, Gatersleben, Germany) for kindly providing the Morex genomic sequences. We thank Dr F. van Eeuwijk and Dr O. Dolstra for useful discussions. We acknowledge Harry Belcram (INRA URGV, France) for helping with the construction of the two BAC libraries. The authors wish to thank the University Malaysia Sarawak, Malaysia for granting the PhD fellowship to FKS Yeo allowing this work at Wageningen University, the Netherlands.

\section{Compliance with ethical standards}

Conflict of interest The authors declare that they have no conflict of interest.

Open Access This article is distributed under the terms of the Creative Commons Attribution 4.0 International License (http://creativecommons.org/licenses/by/4.0/), which permits unrestricted use, distribution, and reproduction in any medium, provided you give appropriate credit to the original author(s) and the source, provide a link to the Creative Commons license, and indicate if changes were made.

\section{References}

Allouis S, Moore G, Bellec A, Sharp R, Rampant PF, Mortimer K, Pateyron S, Foote TN, Griffiths S, Caboche M (2003) Construction and characterisation of a hexaploid wheat (Triticum aestivum L.) BAC library from the reference germplasm'Chinese Spring'. Cereal Res Commun 31:331-338

Almagro L, Gómez Ros LV, Belchi-Navarro S, Bru R, Ros Barceló A, Pedreño MA (2009) Class III peroxidases in plant defence reactions. J Exp Bot 60:377-390 
Antolín-Llovera M, Ried MK, Binder A, Parniske M (2012) Receptor kinase signaling pathways in plant-microbe interactions. Annu Rev Phytopathol 50:451-473

Arru L, Francia E, Pecchioni N (2003) Isolate-specific QTLs of resistance to leaf stripe (Pyrenophora graminea) in the 'Steptoe' $\times$ 'Morex' spring barley cross. Theor Appl Genet 106:668-675

Atienza S, Jafary H, Niks RE (2004) Accumulation of genes for susceptibility to rust fungi for which barley is nearly a nonhost results in two barley lines with extreme multiple susceptibility. Planta 220:71-79

Brugmans B, van der Hulst RGM, Visser RGF, Lindhout P, van Eck HJ (2003) A new and versatile method for the successful conversion of AFLPTM markers into simple single locus markers. Nucleic Acids Res 31:e55

Brugmans B, Hutten RGB, Rookmaker ANO, Visser RGF, Eck HJ (2006) Exploitation of a marker dense linkage map of potato for positional cloning of a wart disease resistance gene. Theor Appl Genet 112:269-277

Chalhoub B, Belcram H, Caboche M (2004) Efficient cloning of plant genomes into bacterial artificial chromosome (BAC) libraries with larger and more uniform insert size. Plant Biotechnol J 2:181-188

Chen X, Niks R, Hedley P, Morris J, Druka A, Marcel T, Vels A, Waugh R (2010) Differential gene expression in nearly isogenic lines with QTL for partial resistance to Puccinia hordei in barley. BMC Genom 11:629

Clarke L, Carbon J (1976) A colony bank containing synthetic CoI EI hybrid plasmids representative of the entire E. coli genome. Cell 9:91-99

Den Herder G, Yoshida S, Antolín-Llovera M, Ried MK, Parniske M (2012) Lotus japonicus E3 ligase SEVEN IN ABSENTIA4 destabilizes the symbiosis receptor-like kinase SYMRK and negatively regulates rhizobial infection. Plant Cell 24:1691-1707

Dros J (1957) The creation and maintenance of two spring barley varieties. Euphytica 6:45-48

Faris JD, Liu Z, Xu SS (2013) Genetics of tan spot resistance in wheat. Theor Appl Genet 126:2197-2217

Fu H, Dooner HK (2002) Intraspecific violation of genetic colinearity and its implications in maize. Proc Natl Acad Sci USA 99:9573-9578

Fu D, Uauy C, Distelfeld A, Blechl A, Epstein L, Chen X, Sela H, Fahima T, Dubcovsky J (2009) A kinase-START gene confers temperature-dependent resistance to wheat stripe rust. Science 323:1357-1360

Fukuoka S, Saka N, Koga H, Ono K, Shimizu T, Ebana K, Hayashi N, Takahashi A, Hirochika H, Okuno K, Yano M (2009) Loss of function of a proline-containing protein confers durable disease resistance in rice. Science 325:998-1001

Giese H, Holm-Jensen AG, Jensen HP, Jensen J (1993) Localization of the Laevigatum powdery mildew resistance gene to barley chromosome 2 by the use of RFLP markers. Theor Appl Genet 85:897-900

González AM, Marcel TC, Kohutova Z, Stam P, van der Linden CG, Niks RE (2010) Peroxidase profiling reveals genetic linkage between peroxidase gene clusters and basal host and non-host resistance to rusts and mildew in barley. PLoS One 5:e10495

Gore MA, Chia J, Elshire RJ, Sun Q, Ersoz ES, Hurwitz BL, Peiffer JA, McMullen MD, Grills GS, Ross-Ibarra J, Ware DH, Buckler ES (2009) A first-generation haplotype map of maize. Science 326:1115-1117

Hayashi N, Inoue H, Kato T, Funao T, Shirota M, Shimizu T, Kanamori H, Yamane H, Hayano-Saito Y, Matsumoto T, Yano M, Takatsuji H (2010) Durable panicle blast-resistance gene $\mathrm{Pb} 1$ encodes an atypical CC-NBS-LRR protein and was generated by acquiring a promoter through local genome duplication. Plant $\mathbf{J}$ 64:498-510

Hickey LT, Lawson W, Platz GJ, Dieters M, Franckowiak J (2012) Origin of leaf rust adult plant resistance gene Rph20 in barley. Genome 55:396-399

Hu G, Fearon ER (1999) Siah-1 N-terminal RING domain is required for proteolysis function, and C-terminal sequences regulate oligomerization and binding to target proteins. Mol Cell Biol 19:724-732

Hückelhoven R, Kogel K-H (2003) Reactive oxygen intermediates in plant-microbe interactions: who is who in powdery mildew resistance? Planta 216:891-902

Isidore E, Scherrer B, Bellec A, Budin K, Faivre P, Waugh R, Keller B, Caboche M, Feuillet C, Chalhoub B (2005) Direct targeting and rapid isolation of BAC clones spanning a defined chromosome region. Funct Integr Genom 5:97-103

Jafary H, Szabo LJ, Niks RE (2006) Innate nonhost immunity in barley to different heterologous rust fungi is controlled by sets of resistance genes with different and overlapping specificities. Mol Plant Microbe Interact 19:1270-1279

Jafary H, Albertazzi G, Marcel TC, Niks RE (2008) High diversity of genes for nonhost resistance of barley to heterologous rust fungi. Genetics 178:2327-2339

Janda J, Bartoš J, Šafář J, Kubaláková M, Valárik M, Č́íhalíková J, Šimková H, Caboche M, Sourdille P, Bernard M, Chalhoub B, Doležel J (2004) Construction of a subgenomic BAC library specific for chromosomes 1D, 4D and 6D of hexaploid wheat. Theor Appl Genet 109:1337-1345

Johnston PA, Niks RE, Meiyalaghan V, Blanchet E, Pickering R (2013) Rph22: mapping of a novel leaf rust resistance gene introgressed from the non-host Hordeum bulbosum L. into cultivated barley (Hordeum vulgare L.). Theor Appl Genet 126:1613-1625

Jones JDG, Dangl JL (2006) The plant immune system. Nature 444:323-329

Kraakman ATW, Martínez F, Mussiraliev B, Eeuwijk FA, Niks RE (2006) Linkage disequilibrium mapping of morphological, resistance, and other agronomically relevant traits in modern spring barley cultivars. Mol Breed 17:41-58

Krattinger SG, Lagudah ES, Spielmeyer W, Singh RP, Huerta-Espino J, McFadden H, Bossolini E, Selter LL, Keller B (2009) A putative $\mathrm{ABC}$ transporter confers durable resistance to multiple fungal pathogens in wheat. Science 323:1360-1363

Kurtz S, Phillippy A, Delcher A, Smoot M, Shumway M, Antonescu C, Salzberg S (2004) Versatile and open software for comparing large genomes. Genome Biol 5:R12

Leroy P, Guilhot N, Sakai H, Bernard A, Choulet F, Theil S, Reboux S, Amano N, Flutre T, Pelegrin C, Ohyanagi H, Seidel M, Giacomoni F, Reichstadt M, Alaux M, Gicquello E, Legeai F, Cerutti L, Numa H, Tanaka T, Mayer K, Itoh T, Quesneville H, Feuillet C (2012) TriAnnot: a versatile and high performance pipeline for the automated annotation of plant genomes. Front Plant Sci 3:5. doi:10.3389/fpls.2012.00005

Ma Z, Weining S, Sharp PJ, Liu C (2000) Non-gridded library: a new approach for BAC (bacterial artificial chromosome) exploitation in hexaploid wheat (Triticum aestivum). Nucleic Acids Res 28:e106

Mago R, Tabe L, Vautrin S, Šimková H, Kubaláková M, Upadhyaya N, Berges H, Kong X, Breen J, Doležel J, Appels R, Ellis JG, Spielmeyer W (2014) Major haplotype divergence including multiple germin-like protein genes, at the wheat $\mathrm{Sr} 2$ adult plant stem rust resistance locus. BMC Plant Biol 14:379

Manosalva PM, Davidson RM, Liu B, Zhu X, Hulbert SH, Leung H, Leach JE (2009) A germin-like protein gene family functions as a complex quantitative trait locus conferring broad-spectrum disease resistance in rice. Plant Physiol 149:286-296 
Marcel TC, Aghnoum R, Durand J, Varshney RK, Niks RE (2007a) Dissection of the barley 2L1. 0 region carrying the 'Laevigatum' quantitative resistance gene to leaf rust using near-isogenic lines (NIL) and subNIL. Mol Plant Microbe Interact 20:1604-1615

Marcel TC, Varshney RK, Barbieri M, Jafary H, de Kock MJD, Graner A, Niks RE (2007b) A high-density consensus map of barley to compare the distribution of QTLs for partial resistance to Puccinia hordei and of defence gene homologues. Theor Appl Genet 114:487-500

Marcel TC, Gorguet B, Ta MT, Kohutova Z, Vels A, Niks RE (2008) Isolate specificity of quantitative trait loci for partial resistance of barley to Puccinia hordei confirmed in mapping populations and near-isogenic lines. New Phytol 177:743-755

Mayer KFX, Martis M, Hedley PE, Šimková H, Liu H, Morris JA, Steuernagel B, Taudien S, Roessner S, Gundlach H, Kubaláková M, Suchánková P, Murat F, Felder M, Nussbaumer T, Graner A, Salse J, Endo T, Sakai H, Tanaka T, Itoh T, Sato K, Platzer M, Matsumoto T, Scholz U, Doležel J, Waugh R, Stein N (2011) Unlocking the barley genome by chromosomal and comparative genomics. Plant Cell 23:1249-1263

Mbengue M, Camut S, de Carvalho-Niebel F, Deslandes L, Froidure S, Klaus-Heisen D, Moreau S, Rivas S, Timmers T, Hervé C, Cullimore J, Lefebvre B (2010) The Medicago truncatula E3 ubiquitin ligase PUB1 interacts with the LYK3 symbiotic receptor and negatively regulates infection and nodulation. Plant Cell 22:3474-3488

Meyers BC, Kaushik S, Nandety RS (2005) Evolving disease resistance genes. Curr Opin Plant Biol 8:129-134

Niks RE (1983a) Comparative histology of partial resistance and the nonhost reaction to leaf rust pathogens in barley and wheat seedlings. Phytopathology 73:60-64

Niks RE (1983b) Haustorium formation by Puccinia hordei in leaves of hypersensitive, partially resistant, and nonhost plant genotypes. Phytopathology 73:64-66

Niks RE (1987) Nonhost plant species as donors for resistance to pathogens with narrow host range I. Determination of nonhost status. Euphytica 36:841-852

Niks RE, Marcel TC (2009) Nonhost and basal resistance: how to explain specificity? New Phytol 182:817-828

Niks RE, Fernandez E, Bv Haperen, Bekele Aleye B, Martinez F (2000) Specificity of QTLs for partial and non-host resistance of barley to leaf rust fungi. Acta Phytopathol Entomol Hun 35:13-21

Niks RE, Parlevliet JE, Lindhout P, Bai Y (2011) Breeding crops with resistance to diseases and pests. Wageningen Academic Publishers, Wageningen, $\mathrm{p} 198$

Nilmalgoda SD, Cloutier S, Walichnowski AZ (2003) Construction and characterization of a bacterial artificial chromosome (BAC) library of hexaploid wheat (Triticum aestivum L.) and validation of genome coverage using locus-specific primers. Genome 46:870-878

Noël L, Moores TL, van der Biezen EA, Parniske M, Daniels MJ, Parker JE, Jones JDG (1999) Pronounced intraspecific haplotype divergence at the RPP5 complex disease resistance locus of Arabidopsis. Plant Cell 11:2099-2111

Parlevliet JE, van Ommeren A (1975) Partial resistance of barley to leaf rust, Puccinia hordei. II. Relationship between field trials, micro plot tests and latent period. Euphytica 24:293-303

Peterson DG, Tomkins JP, Frisch DA, Wing RA, Paterson AH (2000) Construction of plant bacterial artificial chromosome (BAC) libraries: an illustrated guide. J Agric Genom 5:1-100

Qi X, Niks RE, Stam P, Lindhout P (1998) Identification of QTLs for partial resistance to leaf rust (Puccinia hordei) in barley. Theor Appl Genet 96:1205-1215
Qi X, Jiang G, Chen W, Niks RE, Stam P, Lindhout P (1999) Isolatespecific QTLs for partial resistance to Puccinia hordei in barley. Theor Appl Genet 99:877-884

Rodriguez MCS, Petersen M, Mundy J (2010) Mitogen-activated protein kinase signaling in plants. Annu Rev Plant Biol 61:621-649

Saisho D, Myoraku E, Kawasaki S, Sato K, Takeda K (2007) Construction and characterization of a bacterial artificial chromosome (BAC) library from the Japanese malting barley variety 'Haruna Nijo'. Breed Sci 57:29-38

Sakata K, Nagamura Y, Numa H, Antonio BA, Nagasaki H, Idonuma A, Watanabe W, Shimizu Y, Horiuchi I, Matsumoto T, Sasaki T, Higo K (2002) RiceGAAS: an automated annotation system and database for rice genome sequence. Nucleic Acids Res 30:98-102

Salvaudon L, Giraud T, Shykoff JA (2008) Genetic diversity in natural populations: a fundamental component of plant-microbe interactions. Curr Opin Plant Biol 11:135-143

Sambrook J, Fritsch EF, Maniatis T (1989) Molecular cloning, a laboratory manual, 2nd edn. Cold Spring Harbor Laboratory Press, Cold Spring Harbor, p 626

Scherrer B, Isidore E, Klein P, Kim J-S, Bellec A, Chalhoub B, Keller B, Feuillet C (2005) Large intraspecific haplotype variability at the $R p h 7$ locus results from rapid and recent divergence in the barley genome. Plant Cell 17:361-374

Schulte D, Ariyadasa R, Shi B, Fleury D, Saski C, Atkins M, Wu C, Graner A, Langridge P, Stein N (2011) BAC library resources for map-based cloning and physical map construction in barley (Hordeum vulgare L.). BMC Genom 12:247

Shen J, Araki H, Chen L, Chen J, Tian D (2006) Unique evolutionary mechanism in R-genes under the presence/absence polymorphism in Arabidopsis thaliana. Genetics 172:1243-1250

Shi BJ, Sutton T, Collins NC, Pallotta M, Langridge P (2010) Construction of a barley bacterial artificial chromosome library suitable for cloning genes for boron tolerance, sodium exclusion and high grain zinc content. Plant Breed 129:291-296

The International Barley Genome Sequencing Consortium (2012) A physical, genetic and functional sequence assembly of the barley genome. Nature 491:711-716

Thiel T, Michalek W, Varshney R, Graner A (2003) Exploiting EST databases for the development and characterization of genederived SSR-markers in barley (Hordeum vulgare L.). Theor Appl Genet 106:411-422

Trujillo M, Troeger M, Niks RE, Kogel K, Hückelhoven R (2004) Mechanistic and genetic overlap of barley host and non-host resistance to Blumeria graminis. Mol Plant Pathol 5:389-396

van Berloo R, Aalbers H, Werkman A, Niks R (2001) Resistance QTL confirmed through development of QTL-NILs for barley leaf rust resistance. Mol Breed 8:187-195

Varshney R, Marcel TC, Ramsay L, Russell J, Röder MS, Stein N, Waugh R, Langridge P, Niks RE, Graner A (2007) A high density barley microsatellite consensus map with 775 SSR loci. Theor Appl Genet 114:1091-1103

Xia Z, Wu H, Watanabe S, Harada K (2014) Construction and targeted retrieval of specific clone from a non-gridded soybean bacterial artificial chromosome library. Anal Biochem 444:38-40

Yeo FKS, Hensel G, Vozábová T, Martin-Sanz A, Marcel TC, Kumlehn J, Niks RE (2014) Golden SusPtrit: a genetically well transformable barley line for studies on the resistance to rust fungi. Theor Appl Genet 127:325-337

Yu Y, Tomkins JP, Waugh R, Frisch DA, Kudrna D, Kleinhofs A, Brueggeman RS, Muehlbauer GJ, Wise RP, Wing RA (2000) A bacterial artificial chromosome library for barley (Hordeum vulgare L.) and the identification of clones containing putative resistance genes. Theor Appl Genet 101:1093-1099 